3. F. T. Birtel, Banach algebras of multipliers, Duke Math. J. 28 (1961), 203-212.

4. - Isomorphisms and isometric multipliers, Proc. Amer. Math. Soc. 13 (1962), 204-210.

5. W. F. Eberlein, Characterizations of Fourier-Stieltjes transforms, Duke Math. J. 22 (1955), 465-468. $51-67$.

6. I. Glicksberg, Convolution semi-groups of measures, Pacific J. Math. 9 (1959),

7. L. H. Loomis, An introduction to abstract harmonic analysis, Van Nostrand, New York, 1953.

UNiversity of Notre Dame AND

YALE UNIVERSITY

\title{
A GENERAL THEORY OF $k$-PLACE STROKE FUNCTIONS IN 2-VALUED LOGIC
}

ATWELL R. TURQUETTE

Let $O$ be a 2 -valued operator which may be applied to $k(k \geqq 1)$ statements $P_{1}, \cdots, P_{k}$ to generate a new statement $O\left(P_{1}, \cdots, P_{k}\right)$. Let $o\left(p_{1}, \cdots, p_{k}\right)$ denote the truth-value function associated with $O\left(P_{1}, \cdots, P_{k}\right)$. We will assume that $o\left(p_{1}, \cdots, p_{k}\right)$ is defined by the standard truth table which is associated with the statement $O\left(P_{1}, \cdots, P_{k}\right)$ when 1 denotes "true" and 2 denotes "false." $A$ set of operators $\left\{O_{1}, \cdots, O_{t}\right\}$ will be called functionally complete if a statement calculus based on $O_{1}, \cdots, O_{t}$ is functionally complete. If the set $\{O\}$ is functionally complete, then $O$ will be called $a k$-place stroke and $O\left(P_{1}, \cdots, P_{k}\right)$ will be called $a k$-place stroke function. If $k=2$, there are exactly two $k$-place stroke functions; namely, the well-known $\uparrow\left(P_{1}, P_{2}\right)$ and $\downarrow\left(P_{1}, P_{2}\right)$ which are such that $\uparrow\left(p_{1}, p_{2}\right)$ $=3-\max \left(p_{1}, p_{2}\right)$ and $\downarrow\left(p_{1}, p_{2}\right)=3-\min \left(p_{1}, p_{2}\right)$. The purpose of this paper is to give a general method for constructing and calculating the number of $k$-place stroke functions for any given $k(1,2, \cdots, N)$. If $k=1$, then the set of $k$-place stroke functions is null. Hence, the present problem of generating $k$-place stroke functions becomes interesting only when $k \geqq 3$.

If a standard truth table is constructed for $O\left(P_{1}, \cdots, P_{k}\right)$, it will contain $2^{k}$ rows, and the $i$ th row will correspond to the $i$ th term in the lexicographical ordering of the sets of truth values $\left[v_{1}, \cdots, v_{k}\right]$ which are assigned to the arguments $P_{1}, \cdots, P_{k}$. Let $X_{i}$ denote the truth value of $O\left(P_{1}, \cdots, P_{k}\right)$ in the $i$ th row of a standard truth table for $O\left(P_{1}, \cdots, P_{k}\right)$. The truth values of $O\left(P_{1}, \cdots, P_{k}\right)$ which appear

Received by the editors August 8, 1961. 
in its standard table are then $X_{1}, X_{2}, \cdots, X_{2^{k}}$ in order. We will call $\left[X_{1}, X_{2}, \cdots, X_{2^{k}}\right]$ the value column of $O\left(P_{1}, \cdots, P_{k}\right)$.

By a result of Post [1], $O$ is a $k$-place stroke only if the value column of $O\left(P_{1}, \cdots, P_{k}\right)$ is such that $X_{1}=2$ and $X_{2^{k}}=1$, where 1 denotes "true" and 2 denotes "false." We thus restrict attention to value columns of the form $\left[2, X_{2}, X_{3}, \cdots, X_{2^{k}-1}, 1\right]$. If in such a column $X_{2}=X_{2^{k}-1}$, then $O$ is a $k$-place stroke, since $\uparrow\left(p_{1}, p_{2}\right)$ can be defined when $X_{2}=1$ and $\downarrow\left(p_{1}, p_{2}\right)$ can be defined when $X_{2}=2$. To this end, use can be made of the definition schema $O\left(P v_{1}^{0}, P v_{2}^{0}, \cdots, P v_{k}^{0}\right)$ where $o\left(v_{1}^{0}, \quad v_{2}^{0}, \cdots, \quad v_{k}^{0}\right)=X_{2}$. For example, $\uparrow\left(\begin{array}{ll}P_{1}, & \left.P_{2}\right)\end{array}\right.$ $={ }_{D F} O\left(P_{1}, P_{1}, \cdots, P_{2}\right)$ if $X_{2}=1$, and $\downarrow\left(P_{1}, P_{2}\right)={ }_{D F} O\left(P_{1}, P_{1}, \cdots, P_{2}\right)$ if $X_{2}=2$. But this means that $2^{2^{k}-3} k$-place strokes exist with value columns of the form $\left[2, X_{2}, X_{3}, \cdots, X_{2^{k}-2}, X_{2}, 1\right]$. If $X_{2} \neq X_{2^{k}-1}$, then $X_{2^{k}-1}=3-X_{2}$ and we write $X_{2^{k}-1}=\sim X_{2}$ or $X_{2}=\sim X_{2^{k}-1}$ since $X_{j}\left(j=1, \cdots, 2^{k}\right)=\left\{\begin{array}{l}1 \\ 2\end{array}\right.$. Assume $X_{2} \neq X_{2^{k}-1}$. If $X_{3}=X_{2^{k}-2}$, the value column of $O$ is of the form $\left[2, X_{2}, X_{3}, X_{4}, \cdots, X_{2^{k}-3}, X_{3}, \sim X_{2}, 1\right]$, and $\uparrow\left(p_{1}, p_{2}\right)$ can be defined if $X_{3}=1$ and $\downarrow\left(p_{1}, p_{2}\right)$ can be defined if $X_{3}=2$. As indicated above, if $X_{3}=1, \quad \uparrow\left(P_{1}, P_{2}\right)$ $={ }_{D F} O\left(P_{1}, \cdots, P_{1}, P_{2}, P_{1}\right)$ and the same schema can be used to define $\downarrow\left(P_{1}, P_{2}\right)$ when $X_{3}=2$. Thus, $2^{2^{k}-4} k$-place strokes exist with value columns of the form $\left[2, X_{2}, X_{3}, X_{4}, \cdots, X_{2^{k}-3}, X_{3}, \sim X_{2}, 1\right]$. This means that we now have $2^{2^{k}-3}+2^{2^{k}-4}$ distinct $k$-place stroke functions. If $X_{3} \neq X_{2^{k}-2}$, then first consider the case where $X_{4}=X_{2^{k}-3}$. This gives rise to value columns of the form

$$
\left[2, X_{2}, X_{3}, X_{4}, X_{6}, \cdots, X_{2^{k}-4}, X_{4}, \sim X_{3}, \sim X_{2}, 1\right]
$$

from which $2^{2^{k}-5}$ new $k$-place strokes can be defined to give $2^{2^{k}-a}$ $+2^{2^{k}-4}+2^{2^{k}-5}$ distinct $k$-place stoke functions. Then consider the case where $X_{4} \neq X_{2^{k}-3}$ and continue as before. By repeating this process, a point will be reached where the value column of $O\left(P_{1}, \cdots, P_{k}\right)$ is of the form

$$
\left[2, X_{2}, \cdots, X_{2^{k-1}-1}, X_{2^{k-1}}, X_{2^{k-1}}, \sim X_{2^{k-1}-1}, \cdots, \sim X_{2}, 1\right]
$$

and gives rise to $2^{2^{k-1-1}}$ new $k$-place strokes for a total of $2^{2^{k}-3}$ $+2^{2^{k}-4}+2^{2^{k}-5}+\cdots+2^{2^{k-1}-1} k$-place stroke functions.

The case analysis will be exhaustive if we now take $X_{2^{k-1}} \neq X_{2^{k-1}+1}$ and consider value columns of the form

$$
\left[2, X_{2}, \cdots, X_{2^{k-1}}, \sim X_{2^{k-1}}, \cdots, \sim X_{2}, 1\right] .
$$

If $O\left(P_{1}, \cdots, P_{k}\right)$ has such a value column, then it is self-dual in Post's sense [2], and from one of his major results [3], it follows that the set $\{O\}$ is incomplete and $O$ is not a $k$-place stroke. Hence, no new stroke functions are added by this case. 
In summary, we have the following Theorem:

The number of distinct 2-valued $k$-place $(k \geqq 2)$ stroke functions is given by the finite sum $2^{2^{k}-3}+2^{2^{k}-4}+2^{2^{k}-5}+\cdots+2^{2^{k-1}-1}$, and these $k$-place stroke functions can be constructed in order by first filling in the value column $\left[2, X_{2}, X_{3}, \cdots, X_{2^{k}-2}, X_{2}, 1\right]$ in all possible ways, then filling in the value column $\left[2, X_{2}, X_{3}, X_{4}, \cdots, X_{2^{k}-3}, X_{3}, \sim X_{2}, 1\right]$ in all possible ways, then... , then filling in the value column $\left[2, X_{2}, X_{3}, \cdots, X_{2^{k-1}-1}, X_{2^{k-1}}, X_{2^{k-1}}, \sim X_{2^{k-1}-1}, \cdots, \sim X_{2}, 1\right]$ in all possible ways.

If use is made of Post's result already referred to in [3], then our theorem immediately gives rise to the following Corollary:

The number of $k$-place $(k \geqq 2)$ self-dual Post $\delta$-functions which can be formed from the set $\{0\}$ is $2^{2^{k}-2}-$ (the number of $k$-place stroke functions).

Our theorem and corollary give an easy method for calculating both the number of $k$-place stroke functions and the number of Post $\delta$-functions for a given $k$. For example, the reader may check that for $k=2$, there are two stroke and two $\delta$ functions. For $k=3$, there are 56 stroke and $8 \delta$ functions. For $k=4$, there are 16,256 stroke and 128 $\delta$ functions. As $k$ increases, the number of stroke functions becomes very large, but the method of calculation remains simple.

It seems likely that an analogous method might be devised to calculate the number of $k$-place stroke functions in $M$-valued logic for $M>2$. However, it seems unlikely that the finite sum produced will generate the kind of power series which will make possible an easy method of calculation like the one produced by the present theory for the 2-valued case. In particular, there remains the problem of calculating the number of 2-place stroke functions for $M>2$. As has been suggested recently [4], this problem is probably "closely linked with the unsolved problem of determining the number of all bases of the symmetric group $S_{n}$."

\section{REFERENCES}

1. Emil L. Post, The two-valued iterative systems of mathematical logic, Princeton Univ. Press, Princeton, N. J., 1941, p. 108.

2. —, ibid., pp. 30-31.

3. - ibid., p. 108.

4. Arto Salomaa, On the composition of functions of several variables ranging over a finite set, Ann. Univ. Turku. Ser. AI, Turun Yliopisto, Turku, 1960, p. 34.

UNIVERSITY OF ILLINOIS 